Supporting Information

\title{
Highly efficient and robust nickel phosphides as bifunctional electrocatalysts for overall water-splitting
}

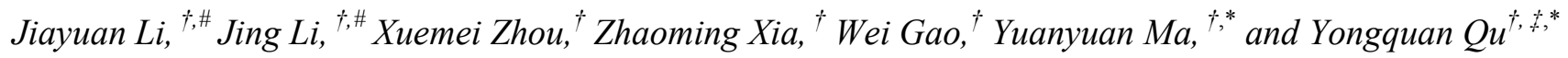

${ }^{\dagger}$ Center for Applied Chemical Research, Frontier Institute of Science and Technology, and State Key Laboratory for Mechanical Behavior of Materials, Xi'an Jiaotong University, Xi'an Jiaotong University, Xi'an, 710049, China.

${ }^{\ddagger}$ MOE Key Laboratory for Nonequilibrium Synthesis and Modulation of Condensed Matter, Xi'an Jiaotong University, Xi'an, 710049, China.

E-mail: yongquan@mail.xjtu.edu.cn,yyma@mail.xjtu.edu.cn

\# Authors contributed equally. 


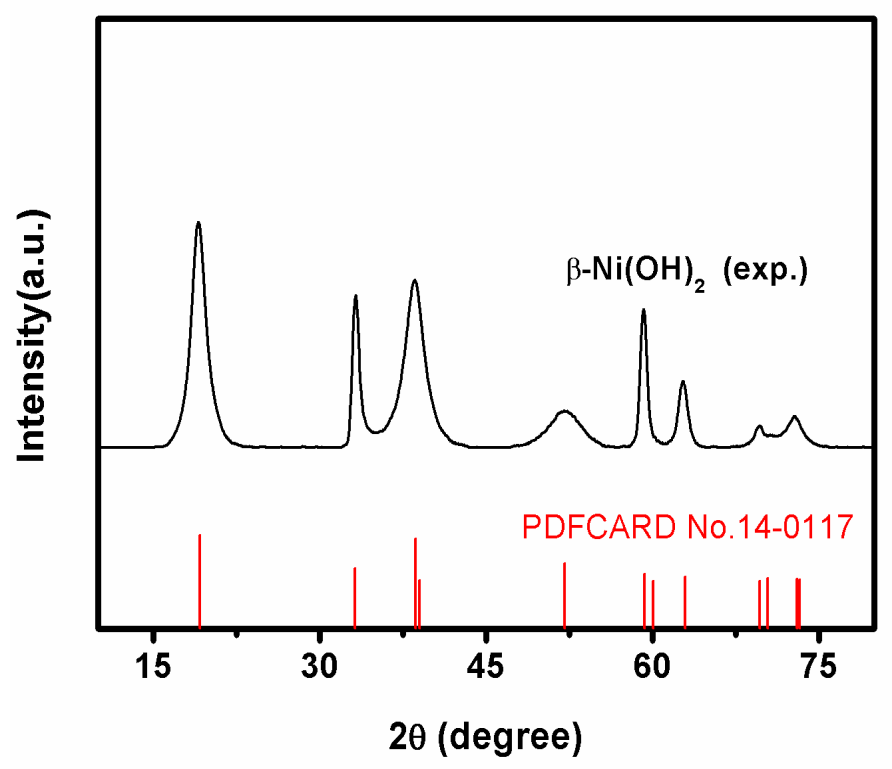

Figure S1. Powder XRD pattern of the as-prepared $\beta-\mathrm{Ni}(\mathrm{OH})_{2}$ nanoplate precursor.
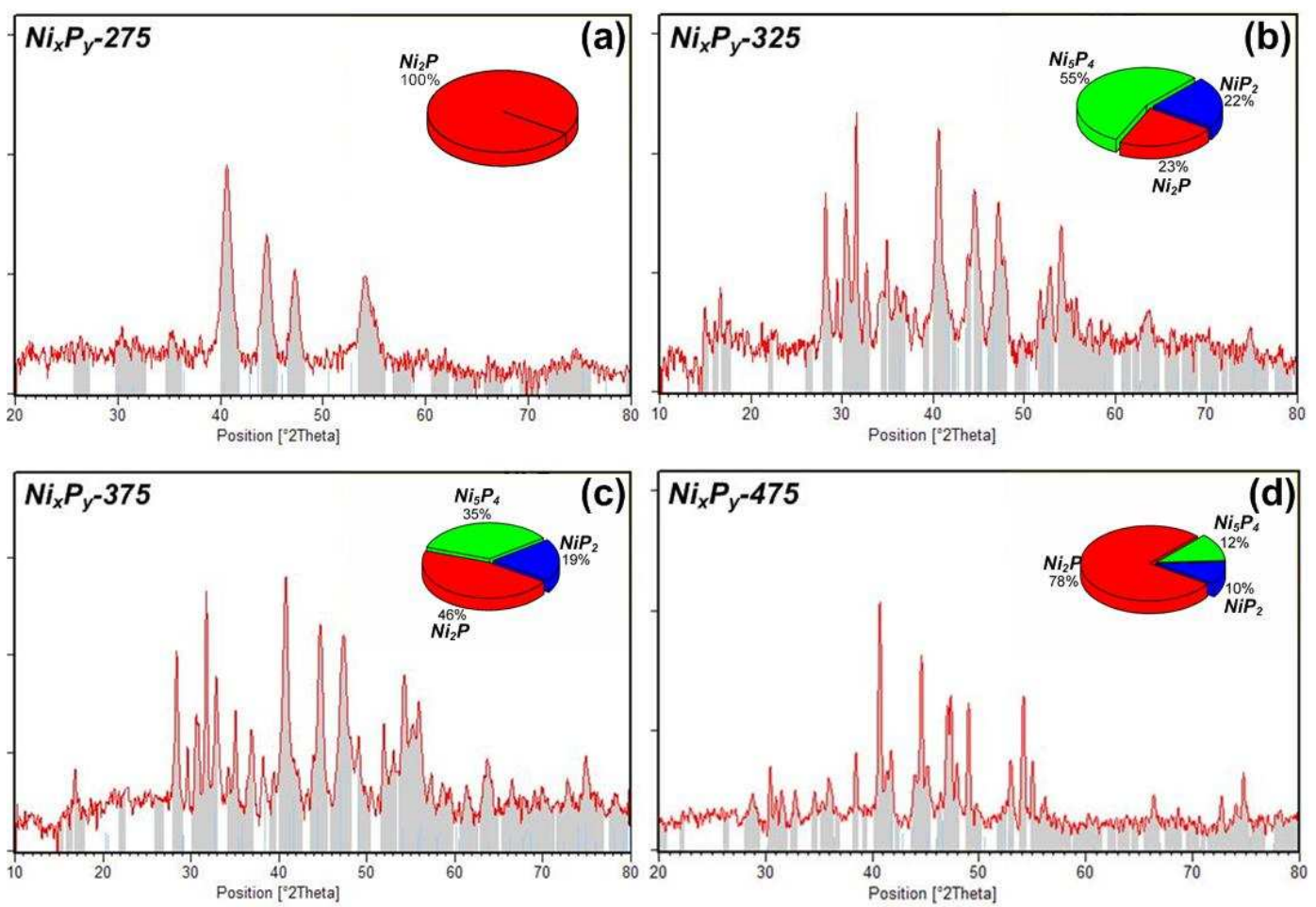

Figure S2. Phase composition analysis of the $\mathrm{Ni}_{\mathrm{x}} \mathrm{P}_{\mathrm{y}}-\mathrm{T}$ catalysts: (a) $\mathrm{Ni}_{\mathrm{x}} \mathrm{P}_{\mathrm{y}}-275$ (b) $\mathrm{Ni}_{\mathrm{x}} \mathrm{P}_{\mathrm{y}}-325$ (c) $\mathrm{Ni}_{\mathrm{x}} \mathrm{P}_{\mathrm{y}^{-}}-375$ (d) $\mathrm{Ni}_{\mathrm{x}} \mathrm{P}_{\mathrm{y}}-475$. 

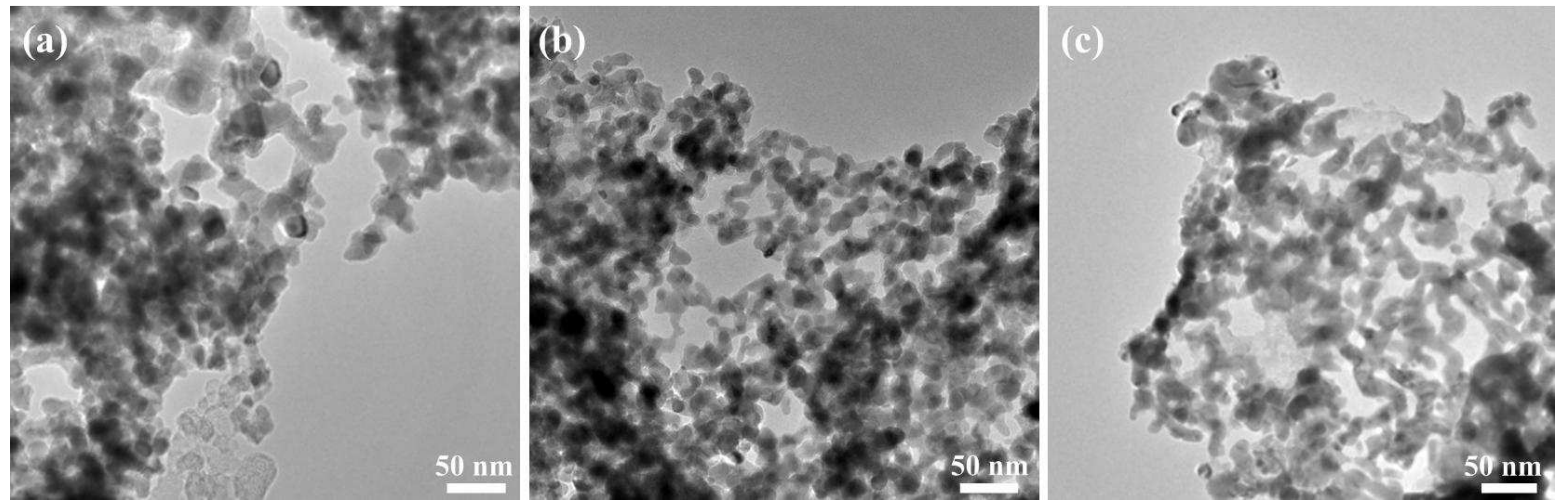

Figure S3. TEM image of as-prepared (a) $\mathrm{Ni}_{\mathrm{x}} \mathrm{P}_{\mathrm{y}}-275$, (b) $\mathrm{Ni}_{\mathrm{x}} \mathrm{P}_{\mathrm{y}}-375$ and (c) $\mathrm{Ni}_{\mathrm{x}} \mathrm{P}_{\mathrm{y}}-475$.

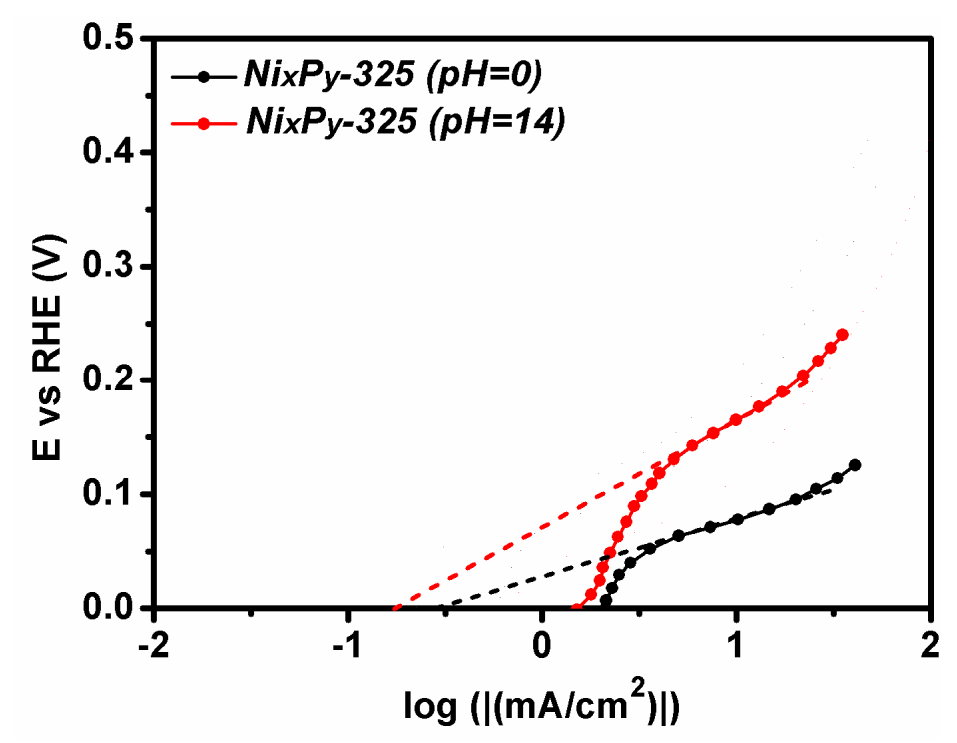

Figure S4. Derived exchange current densities of the $\mathrm{Ni}_{\mathrm{x}} \mathrm{P}_{\mathrm{y}}-325$ catalysts in various $\mathrm{pH}$ environments by applying extrapolation method to the Tafel plots. 


\section{TOF calculation:}

An electrochemical cyclic voltammetry test was employed to quantify the active sites of the $\mathrm{Ni}_{\mathrm{x}} \mathrm{P}_{\mathrm{y}^{-}}-\mathrm{T}$ nanocatalysts in $1.0 \mathrm{M}$ PBS $(\mathrm{pH}=7.0)$ with a potential window from -0.2 to $0.6 \mathrm{~V}$ vs $\mathrm{RHE}$ at a scan rate of $50 \mathrm{mV} / \mathrm{s}$ (Figure S3). ${ }^{1}$ Assuming one electron redox process, the integrated charge over the whole potential range was divided by two. Then, the value was divided by the Faraday constant to get the number of active sites for different samples. The turnover frequency $\left(\mathrm{s}^{-1}\right)$ can be estimated according to this equation ${ }^{2}$ :

$$
\mathrm{TOF}=I / 2 n F
$$

where $I$ represents the current density for different samples during the LSV measurement in $0.5 \mathrm{M} \mathrm{H}_{2} \mathrm{SO}_{4}$, $F$ is the Faraday constant $(\mathrm{C} / \mathrm{mol})$, and $n$ is the number of the active sites (mol) for different samples.

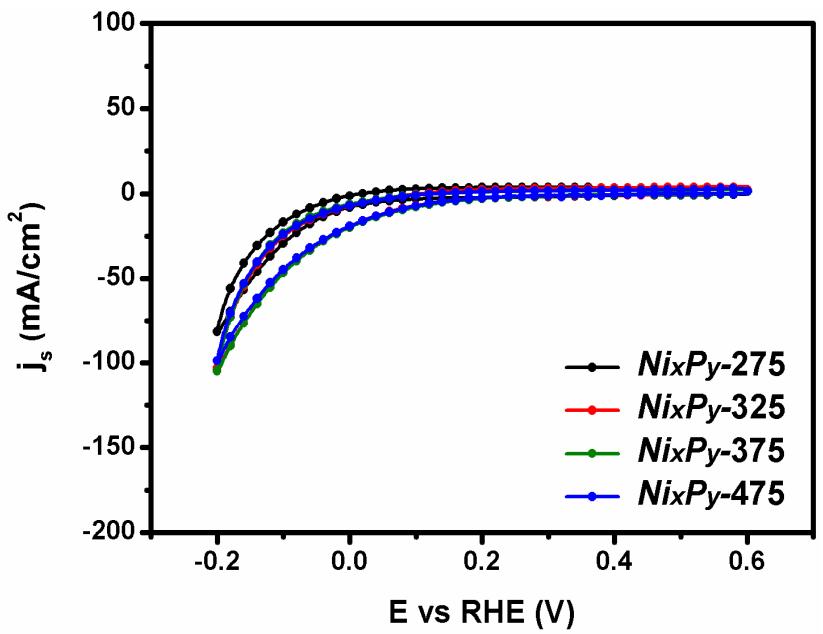

Figure S5. The cyclic voltammetries for the $\mathrm{Ni}_{\mathrm{x}} \mathrm{P}_{\mathrm{y}}-\mathrm{T}$ catalysts in $1.0 \mathrm{M}$ PBS at a scan rate of $50 \mathrm{mV} / \mathrm{s}$.

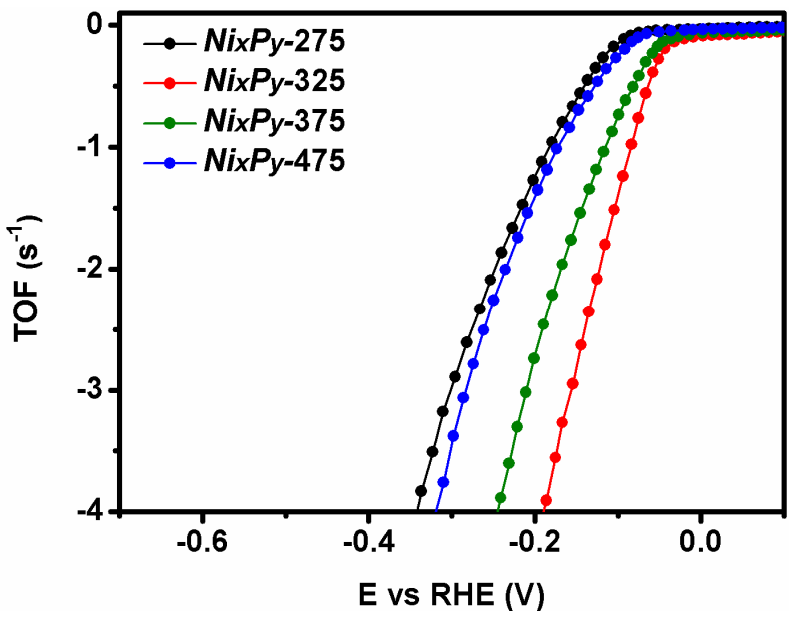

Figure S6. The calculated TOF curves for the $\mathrm{Ni}_{\mathrm{x}} \mathrm{P}_{\mathrm{y}}$-T catalysts in $0.5 \mathrm{M} \mathrm{H}_{2} \mathrm{SO}_{4}$ electrolyte. 


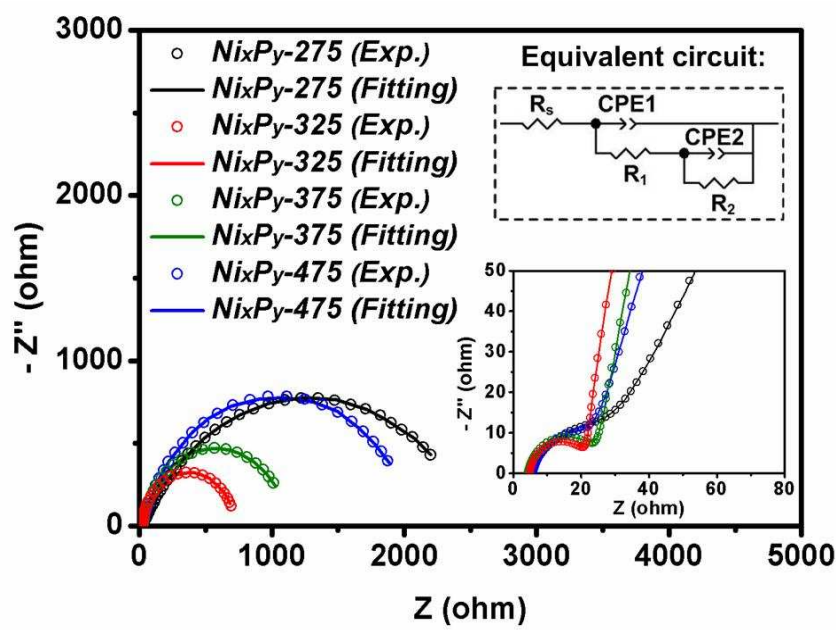

Figure S7. Nyquist plots for the $\mathrm{Ni}_{\mathrm{x}} \mathrm{P}_{\mathrm{y}}-\mathrm{T}$ catalysts in $0.5 \mathrm{M} \mathrm{H}_{2} \mathrm{SO}_{4}$ electrolyte. The scattered symbols represent the experimental results and the solid lines are simulation fitted results. The inset at the top shows the equivalent circuit for the simulation. Electrochemical impedance spectroscopy of the catalysts was carried out on an electrochemistry workstation (AUTOLAB PGSTAT204) under an overpotential of $10 \mathrm{mV}$ within a frequency window of $100 \mathrm{kHz}$ to $0.1 \mathrm{~Hz}$.

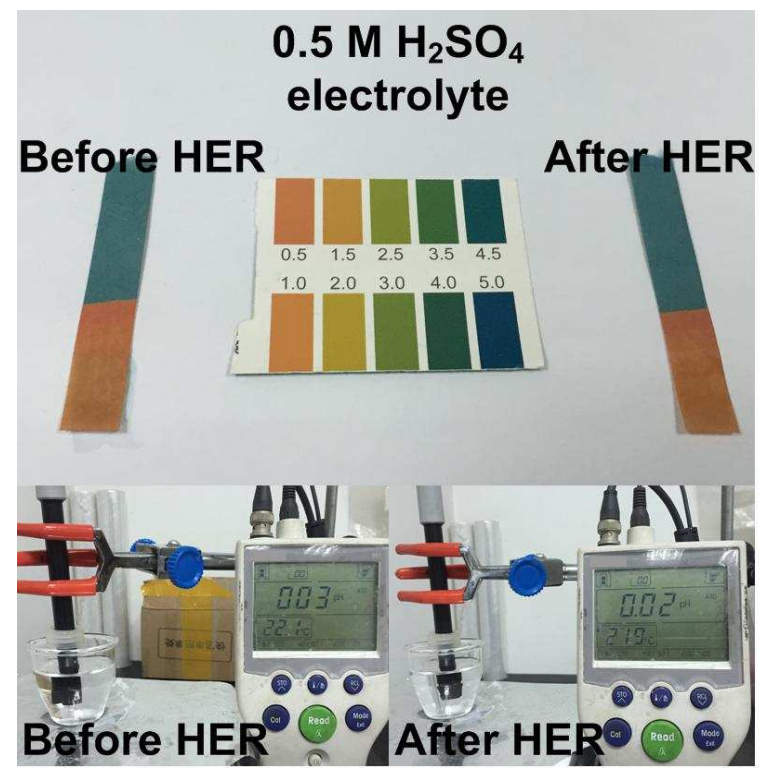

Figure S8. The $\mathrm{pH}$ monitoring in $0.5 \mathrm{M} \mathrm{H}_{2} \mathrm{SO}_{4}$ electrolyte after the HER catalytic durability measurement for the $\mathrm{Ni}_{\mathrm{x}} \mathrm{P}_{\mathrm{y}^{-}}-325$. 


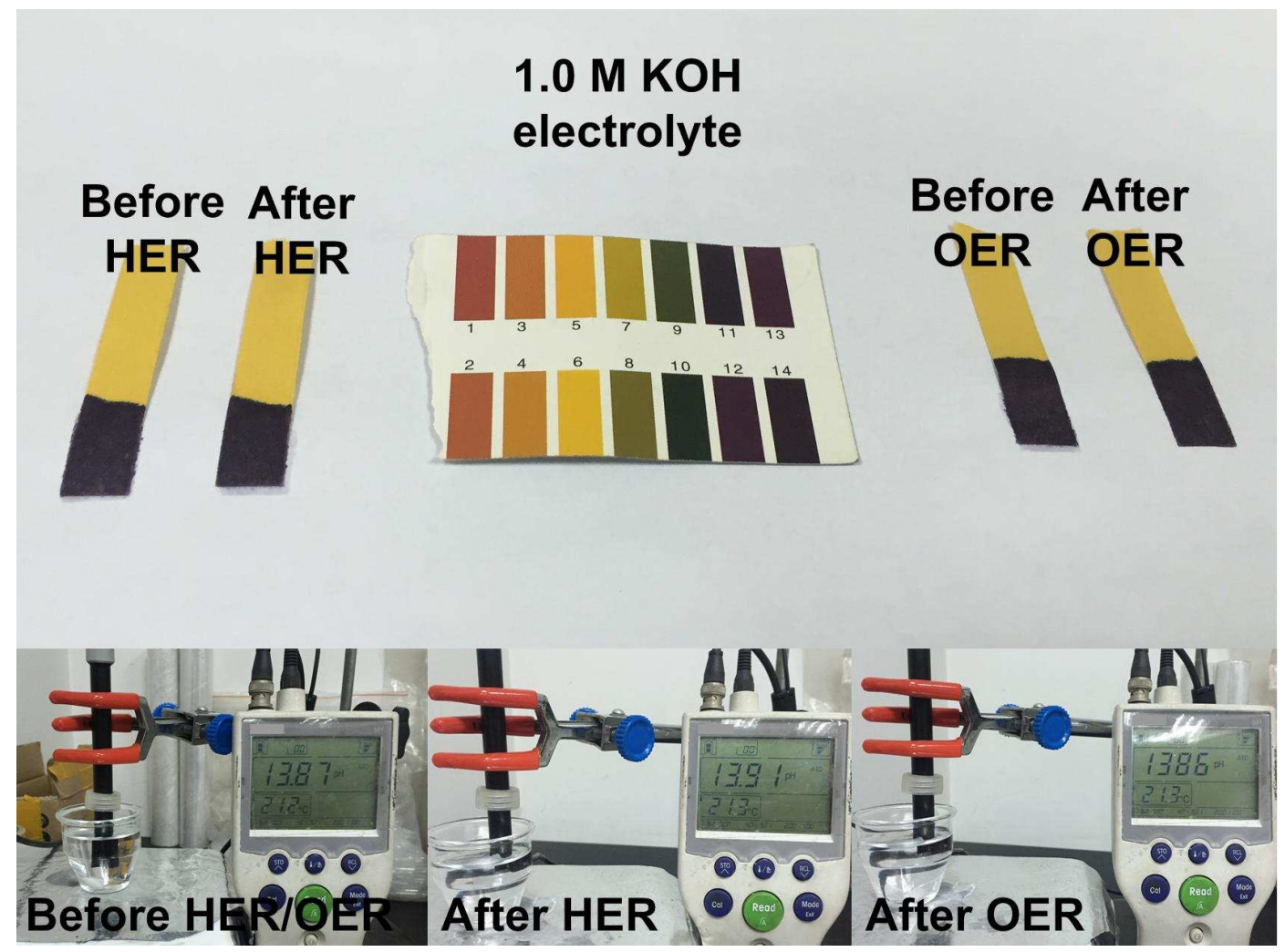

Figure S9. The $\mathrm{pH}$ monitoring in $1.0 \mathrm{M} \mathrm{KOH}$ electrolyte after the HER and OER catalytic durability measurement for the $\mathrm{Ni}_{\mathrm{x}} \mathrm{P}_{\mathrm{y}}-325$.
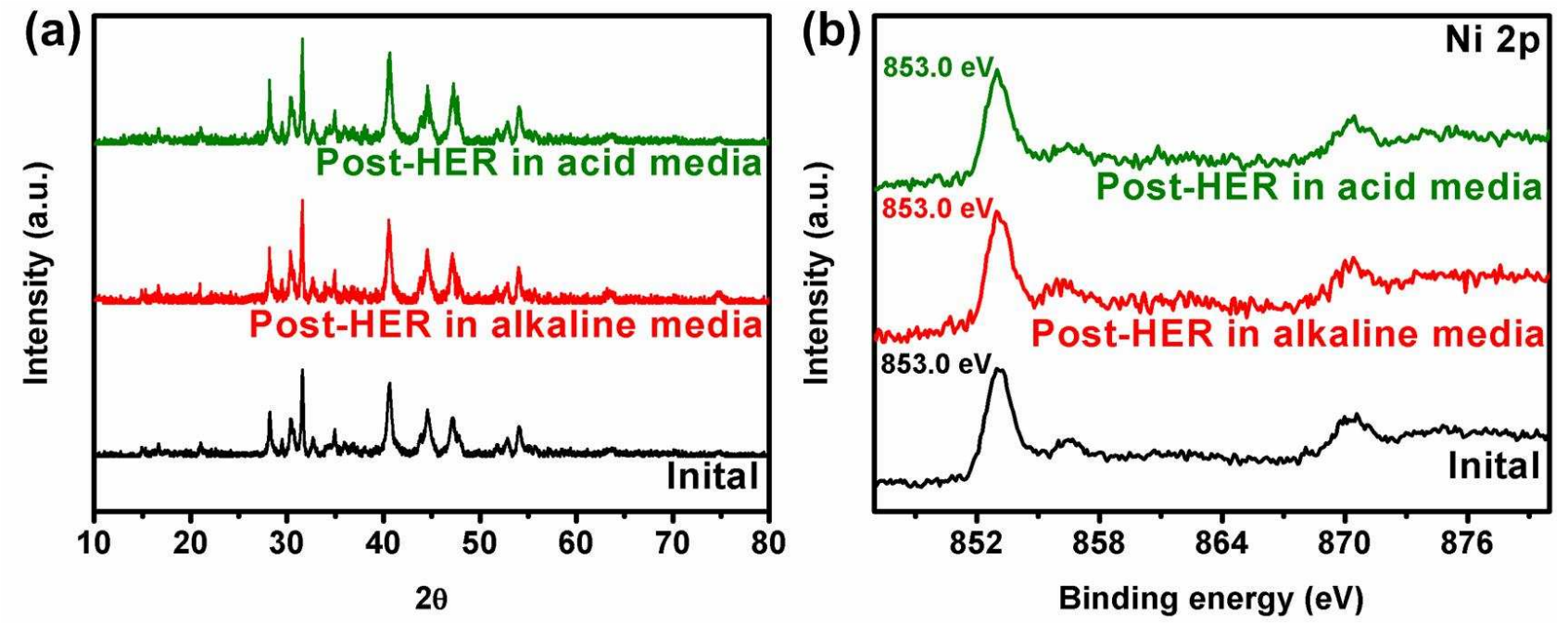

Figure S10. The XRD patterns (a) and XPS spectra of Ni $2 p$ region (b) for the $\mathrm{Ni}_{\mathrm{x}} \mathrm{P}_{\mathrm{y}}-325$ catalyst before and after HER in alkaline $(1.0 \mathrm{M} \mathrm{KOH})$ and acid $\left(0.5 \mathrm{M} \mathrm{H}_{2} \mathrm{SO}_{4}\right)$ media respectively. 

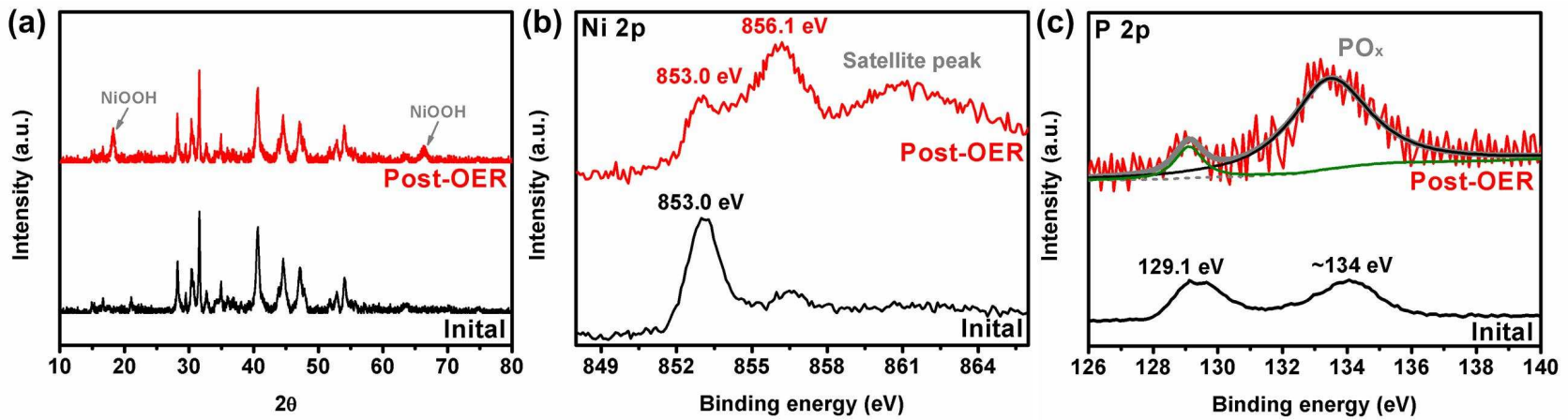

Figure S11. (a) XRD patterns of the $\mathrm{Ni}_{\mathrm{x}} \mathrm{P}_{\mathrm{y}}-325$ catalyst before and after OER in $1.0 \mathrm{M} \mathrm{KOH}$. The XPS spectra of (b) Ni 2p and (c) $\mathrm{P} 2 \mathrm{p}$ region of the $\mathrm{Ni}_{\mathrm{x}} \mathrm{P}_{\mathrm{y}}-325$ catalyst before and after OER in $1.0 \mathrm{M} \mathrm{KOH}$.

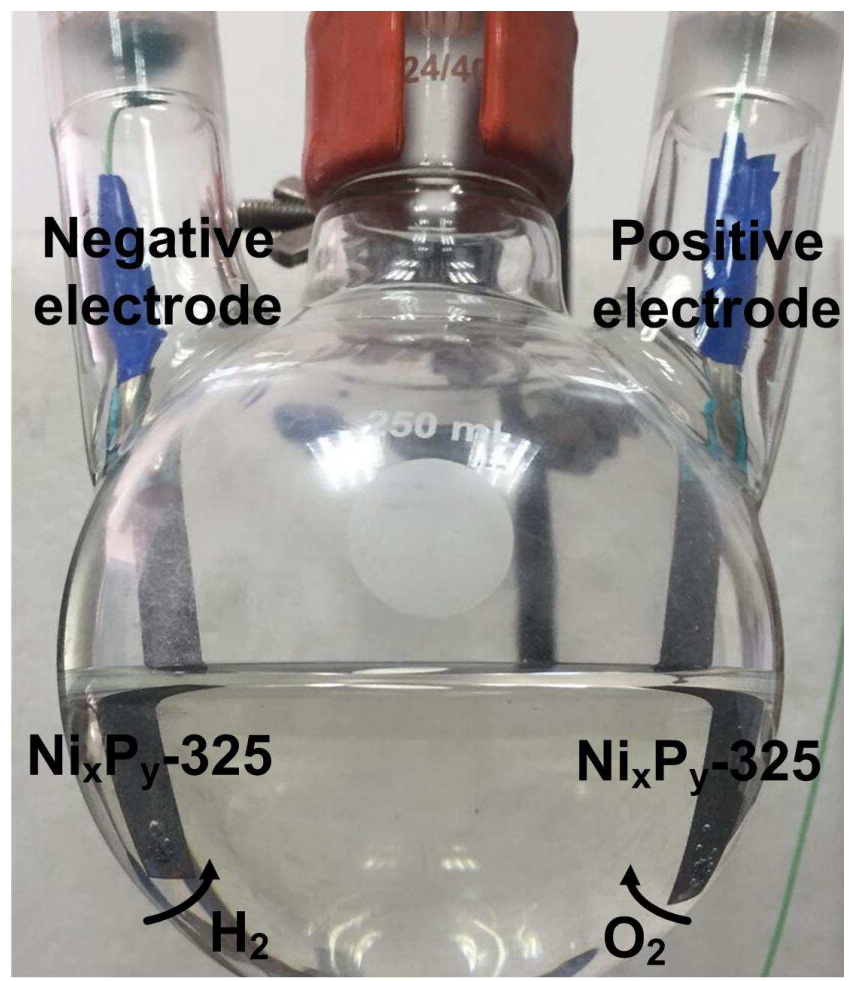

Figure S12. A photograph of the $\mathrm{Ni}_{\mathrm{x}} \mathrm{P}_{\mathrm{y}}-325 \| \mathrm{Ni}_{\mathrm{x}} \mathrm{P}_{\mathrm{y}}-325$ water electrolyzer. 
Table S1. Comparison of HER performance of $\mathrm{Ni}_{\mathrm{x}} \mathrm{P}_{\mathrm{y}}-325$ in acid environment with the nickel- and cobalt-based phosphides.

\begin{tabular}{|c|c|c|c|c|}
\hline Catalyst & $\eta_{20}(\mathrm{mV})$ & Tafel slope (mV/dec) & $\begin{array}{c}\text { Exchange } \\
\text { current density } \\
\left(\mathbf{m A} / \mathrm{cm}^{2}\right)\end{array}$ & References \\
\hline $\begin{array}{c}\mathrm{Ni}_{2} \mathrm{P} \\
\text { nanoparticles }\end{array}$ & 130 & 46 & $3.3 \times 10^{-3}$ & 3 \\
\hline $\begin{array}{c}\mathrm{Ni}_{5} \mathrm{P}_{4} \\
\text { nanocrystals }\end{array}$ & 175 & 42 & 0.057 & 4 \\
\hline $\begin{array}{c}\mathrm{Ni}_{12} \mathrm{P}_{5} \\
\text { nanoparticles }\end{array}$ & 141 & 63 & - & 5 \\
\hline $\begin{array}{c}\mathrm{Ni}_{5} \mathrm{P} 4-\mathrm{Ni}_{2} \mathrm{P} \\
\text { nanosheet }\end{array}$ & 140 & 79.1 & 0.116 & 6 \\
\hline $\mathrm{Ni}_{2} \mathrm{P}$ NPs/Ti & 138 & 60 & - & 7 \\
\hline $\mathrm{Ni}_{2} \mathrm{P} / \mathrm{C}$ & 115 & 54 & - & 8 \\
\hline $\begin{array}{c}\text { CoP } \\
\text { nanoparticles }\end{array}$ & 85 & 50 & 0.14 & 9 \\
\hline $\begin{array}{c}\mathrm{CoP} \\
\text { nanowires }\end{array}$ & 100 & 51 & 0.288 & 2 \\
\hline $\mathrm{CoP} / \mathrm{CNT}$ & 160 & 54 & 0.13 & 10 \\
\hline $\begin{array}{c}\mathrm{Co}_{2} \mathrm{P} \\
\text { nanorods }\end{array}$ & 167 & 51.7 & - & 11 \\
\hline $\begin{array}{c}\mathrm{Ni}_{5} \mathrm{P}_{4} \\
\text { nanocrystals }\end{array}$ & 35 & 33 & - & 12 \\
\hline $\mathrm{Ni}_{5} \mathrm{P}_{4}$ films & $\sim 180$ & $\sim 40$ & - & 13 \\
\hline $\mathrm{Ni}_{\mathbf{x}} \mathbf{P}_{\mathbf{y}}-\mathbf{3 2 5}$ & 62 & 46.1 & 0.275 & This work \\
\hline
\end{tabular}


Table S2. Comparison of HER performance of $\mathrm{Ni}_{\mathrm{x}} \mathrm{P}_{\mathrm{y}}-325$ in acid environment with other non-Pt HER catalysts.

\begin{tabular}{|c|c|c|c|c|}
\hline Catalyst & $\eta_{20}(\mathrm{mV})$ & Tafel slope (mV/dec) & $\begin{array}{c}\text { Exchange } \\
\text { current density } \\
\left(\mathrm{mA} / \mathrm{cm}^{2}\right)\end{array}$ & References \\
\hline $\begin{array}{c}\text { Defect-rich } \mathrm{MoS}_{2} \\
\text { nanosheets }\end{array}$ & 214 & 50 & $8.9 \times 10^{-3}$ & 14 \\
\hline $\mathrm{MoS}_{2} / \mathrm{rGO}$ & 176 & 41 & - & 15 \\
\hline MoS@Au & - & 69 & $9.3 \times 10^{-3}$ & 16 \\
\hline $\mathrm{WS}_{2}$ nanosheets & 275 & 55 & - & 17 \\
\hline MoP & 151 & 54 & 0.086 & 18 \\
\hline $\mathrm{CoSe}_{2}$ & - & 42.4 & $6.5 \times 10^{-5}$ & 19 \\
\hline Ni-Mo nanopower & 80 & - & - & 20 \\
\hline Bulk $\mathrm{Mo}_{2} \mathrm{C}$ & 224 & 56 & $1.3 \times 10^{-3}$ & 21 \\
\hline $\mathrm{Cu}_{2} \mathrm{MoS}_{4}$ & - & 95 & - & 22 \\
\hline $\mathrm{Fe}_{0.9} \mathrm{Co}_{0.1} \mathrm{~S}_{2} / \mathrm{CNT}$ & 120 & 46 & - & 23 \\
\hline FeP nanosheets & $\sim 300$ & 67 & - & 24 \\
\hline $\mathrm{Co}_{0.6} \mathrm{Mo}_{1.4} \mathrm{~N}_{2}$ & 267 & - & $2.3 \times 10^{-4}$ & 25 \\
\hline $\mathbf{N i}_{\mathbf{x}} \mathbf{P}_{\mathbf{y}}-\mathbf{3 2 5}$ & 62 & 46.1 & 0.275 & This work \\
\hline
\end{tabular}


Table S3. Comparison of HER performance of $\mathrm{Ni}_{\mathrm{x}} \mathrm{P}_{\mathrm{y}}-325$ in alkaline environment with other non-precious HER catalysts.

\begin{tabular}{|c|c|c|c|}
\hline Catalyst & $\eta_{20}(\mathrm{mV})$ & Tafel slope (mV/dec) & References \\
\hline CoP nanowire & 335 & 129 & 2 \\
\hline Co-NRCNTs & 476 & - & 26 \\
\hline $\mathrm{Ni}_{0.33} \mathrm{Co}_{0.67} \mathrm{~S}_{2}$ & 129 & 118 & 27 \\
\hline $\mathrm{NiCo}_{2} \mathrm{~S}_{4}$ & 194 & 141 & 28 \\
\hline $\mathrm{CoO}_{\mathrm{x}} / \mathrm{CN}$ & 352 & 114 & 29 \\
\hline $\mathrm{Ni}_{5} \mathrm{P}_{4}$ films & $\sim 180$ & $\sim 53$ & 13 \\
\hline $\mathrm{Ni}_{2} \mathrm{P}$ & 255 & - & 30 \\
\hline $\mathrm{NiFe} \mathrm{LDH} / \mathrm{NF}$ & 250 & - & 31 \\
\hline $\mathrm{MoC}_{\mathrm{x}} / \mathrm{C}$ & $>175$ & 59 & 32 \\
\hline $\mathrm{Ni}_{2} \mathrm{P}$ nanoparticles & 205 & - & 3 \\
\hline $\mathbf{N i}_{\mathbf{x}} \mathbf{P}_{\mathbf{y}}-\mathbf{3 2 5}$ & 160 & 107.3 & This work \\
\hline
\end{tabular}


Table S4. Comparison of OER performance of $\mathrm{Ni}_{\mathrm{x}} \mathrm{P}_{\mathrm{y}}-325$ in alkaline environment with the state-of the-art OER catalysts

\begin{tabular}{|c|c|c|c|}
\hline Catalyst & Electrolyte & $\eta_{10}(\mathrm{mV})$ & References \\
\hline $\mathrm{Ni}-\mathrm{Co}$ binary oxides & $1.0 \mathrm{M} \mathrm{KOH}$ & 325 & 33 \\
\hline $\mathrm{Co}_{0.5} \mathrm{Fe}_{0.5} \mathrm{~S} @ \mathrm{~N}-\mathrm{MC}$ & $1.0 \mathrm{M} \mathrm{KOH}$ & 410 & 34 \\
\hline CoNi SUNOE & $1.0 \mathrm{M} \mathrm{KOH}$ & 450 & 35 \\
\hline NiCo LDH & $1.0 \mathrm{M} \mathrm{KOH}$ & 367 & 36 \\
\hline $\mathrm{Ni}_{\mathrm{x}} \mathrm{Co}_{3-\mathrm{x}} \mathrm{O}_{4} \mathrm{NWs} / \mathrm{Ti}$ & $1.0 \mathrm{M} \mathrm{KOH}$ & 370 & 37 \\
\hline $\mathrm{Co}_{3} \mathrm{O}_{4} / \mathrm{NiCo}_{2} \mathrm{O}_{4} \mathrm{DSNCs}$ & $1.0 \mathrm{M} \mathrm{KOH}$ & 340 & 38 \\
\hline $\mathrm{Ni}_{5} \mathrm{P}_{4}$ films & $1.0 \mathrm{M} \mathrm{KOH}$ & 290 & 13 \\
\hline $\mathrm{Ni}_{2} \mathrm{P}$ & $1.0 \mathrm{M} \mathrm{KOH}$ & 290 & 30 \\
\hline Fe-Ni oxide & $1.0 \mathrm{M} \mathrm{KOH}$ & $>375$ & 39 \\
\hline $\mathrm{CoSe}_{2}$ & $0.1 \mathrm{M} \mathrm{KOH}$ & 320 & 40 \\
\hline NiCo-NS & $1.0 \mathrm{M} \mathrm{KOH}$ & 334 & 41 \\
\hline Co-P film & $1.0 \mathrm{M} \mathrm{KOH}$ & 345 & 42 \\
\hline $\mathrm{MnCo}_{2} \mathrm{O}_{\mathrm{x}}$ & $1.0 \mathrm{M} \mathrm{KOH}$ & $>410$ & 43 \\
\hline $\mathrm{Ni}_{\mathbf{x}} \mathbf{P}_{\mathbf{y}}-\mathbf{3 2 5}$ & $1.0 \mathrm{M} \mathrm{KOH}$ & 320 & This work \\
\hline
\end{tabular}


Table S5. Fitting parameter values of the EIS data of $\mathrm{Ni}_{\mathrm{x}} \mathrm{P}_{\mathrm{y}^{-}}-\mathrm{T}$ catalysts for HER.

\begin{tabular}{ccccccccc}
\hline Sample & $\mathbf{R}_{\mathbf{s}}(\boldsymbol{\Omega})$ & $\mathbf{R}_{\mathbf{1}}(\boldsymbol{\Omega})$ & $\mathbf{n}_{\mathbf{1}}$ & $\mathbf{C P E}_{\mathbf{1}}$ & $\mathbf{R}_{\mathbf{2}}(\boldsymbol{\Omega})$ & $\mathbf{n}_{\mathbf{2}}$ & $\mathbf{C P E}_{\mathbf{2}}$ & $\mathbf{C}_{\mathbf{D L}}(\mathbf{F})$ \\
\hline $\mathrm{Ni}_{\mathrm{x}} \mathrm{P}_{\mathrm{y}}-275$ & 5.5 & 29.9 & 0.89 & $4.5 \times 10^{-5}$ & 2501 & 0.70 & $9.0 \times 10^{-4}$ & $1.81 \times 10^{-5}$ \\
$\mathrm{Ni}_{\mathrm{x}} \mathrm{P}_{\mathrm{y}}-325$ & 4.8 & 17.9 & 0.90 & $5.1 \times 10^{-5}$ & 699 & 0.95 & $8.9 \times 10^{-4}$ & $1.91 \times 10^{-5}$ \\
$\mathrm{Ni}_{\mathrm{x}} \mathrm{P}_{\mathrm{y}}-375$ & 4.2 & 21.8 & 0.87 & $4.1 \times 10^{-5}$ & 1105 & 0.90 & $7.9 \times 10^{-4}$ & $1.94 \times 10^{-5}$ \\
$\mathrm{Ni}_{\mathrm{x}} \mathrm{P}_{\mathrm{y}}-475$ & 5.8 & 24.1 & 0.85 & $8.0 \times 10^{-5}$ & 2047 & 0.83 & $6.1 \times 10^{-4}$ & $1.99 \times 10^{-5}$ \\
\hline
\end{tabular}




\section{References:}

1. Merki, D.; Fierro, S.; Vrubel, H.; Hu, X. Amorphous Molybdenum Sulfide Films as Catalysts for Electrochemical Hydrogen Production in Water. Chem. Sci. 2011, 2, 1262-1267.

2. Tian, J.; Liu, Q.; Asiri, A. M.; Sun, X. Self-Supported Nanoporous Cobalt Phosphide Nanowire Arrays: An Efficient 3D Hydrogen-Evolving Cathode over the Wide Range of pH 0-14. J. Am. Chem. Soc. 2014, 136, 7587-7590.

3. Popczun, E. J.; McKone, J. R.; Read, C. G.; Biacchi, A. J.; Wiltrout, A. M.; Lewis, N. S.; Schaak, R. E. Nanostructured Nickel Phosphide as An Electrocatalyst for the Hydrogen Evolution Reaction. J. Am. Chem. Soc. 2013, 135, 9267-9270.

4. Pan, Y.; Liu, Y.; Zhao, J.; Yang, K.; Liang, J.; Liu, D.; Hu, W.; Liu, D.; Liu, Y.; Liu, C. Monodispersed Nickel Phosphide Nanocrystals with Different Phases: Synthesis, Characterization and Electrocatalytic Properties for Hydrogen Evolution. J. Mater. Chem. A 2015, 3, 1656-1665.

5. Huang, Z.; Chen, Z.; Chen, Z.; Lv, C.; Meng, H.; Zhang, C. $\mathrm{Ni}_{12} \mathrm{P}_{5}$ Nanoparticles as An Efficient Catalyst for Hydrogen Generation via Electrolysis and Photoelectrolysis. ACS Nano 2014, 8, 8121-8129.

6. Wang, X.; Kolen'ko, Y. V.; Bao, X. Q.; Kovnir, K.; Liu, L. One-Step Synthesis of Self-Supported Nickel Phosphide Nanosheet Array Cathodes for Efficient Electrocatalytic Hydrogen Generation. Angew. Chem. Int. Ed. 2015, 54, 8188-8192.

7. Pu, Z.; Liu, Q.; Tang, C.; Asiri, A. M.; Sun, X. Ni 2 P Nanoparticle Films Supported on A Ti Plate as An Efficient Hydrogen Evolution Cathode. Nanoscale 2014, 6, 11031-11034.

8. Bai, Y.; Zhang, H.; Li, X.; Liu, L.; Xu, H.; Qiu, H.; Wang, Y. Novel Peapod-Like $\mathrm{Ni}_{2} \mathrm{P}$ Nanoparticles with Improved Electrochemical Properties for Hydrogen Evolution and Lithium Storage. Nanoscale 2015, 7, 1446-1453.

9. Popczun, E. J.; Read, C. G.; Roske, C. W.; Lewis, N. S.; Schaak, R. E. Highly Active Electrocatalysis of the Hydrogen Evolution Reaction by Cobalt Phosphide Nanoparticles. Angew. Chem. 2014, 126, 5531-5534.

10. Liu, Q.; Tian, J.; Cui, W.; Jiang, P.; Cheng, N.; Asiri, A. M.; Sun, X. Carbon Nanotubes Decorated with CoP Nanocrystals: A Highly Active Non-Noble-Metal Nanohybrid Electrocatalyst for Hydrogen Evolution. Angew. Chem. 2014, 126, 6828-6832.

11. Huang, Z.; Chen, Z.; Chen, Z.; Lv, C.; Humphrey, M. G.; Zhang, C. Cobalt Phosphide Nanorods as An Efficient Electrocatalyst for the Hydrogen Evolution Reaction. Nano Energy 2014, 9, 373-382.

12. Laursen, A.; Patraju, K.; Whitaker, M.; Retuerto, M.; Sarkar, T.; Yao, N.; Ramanujachary, K.; Greenblatt, M.; Dismukes, G. Nanocrystalline $\mathrm{Ni}_{5} \mathrm{P}_{4}$ : A Hydrogen Evolution Electrocatalyst of Exceptional Efficiency in Both Alkaline and Acidic Media. Energy Environ. Sci. 2015, 8, 1027-1034.

13. Ledendecker, M.; Krick Calderón, S.; Papp, C.; Steinrück, H. P.; Antonietti, M.; Shalom, M. The Synthesis of Nanostructured $\mathrm{Ni}_{5} \mathrm{P}_{4}$ Films and Their Use as A Non-Noble Bifunctional Electrocatalyst for Full Water Splitting. Angew. Chem. 2015, 127, 12538-12542.

14. Xie, J.; Zhang, H.; Li, S.; Wang, R.; Sun, X.; Zhou, M.; Zhou, J.; Lou, X. W. D.; Xie, Y. Defect-Rich $\mathrm{MoS}_{2}$ Ultrathin Nanosheets with Additional Active Edge Sites for Enhanced Electrocatalytic Hydrogen Evolution. Adv. Mater. 2013, 25, 5807-5813.

15. Li, Y.; Wang, H.; Xie, L.; Liang, Y.; Hong, G.; Dai, H. MoS 2 Nanoparticles Grown on Graphene: An Advanced Catalyst for the Hydrogen Evolution Reaction. J. Am. Chem. Soc. 2011, 133, 7296-7299.

16. Wang, T.; Liu, L.; Zhu, Z.; Papakonstantinou, P.; Hu, J.; Liu, H.; Li, M. Enhanced Electrocatalytic Activity for Hydrogen Evolution Reaction from Self-Assembled Monodispersed Molybdenum Sulfide Nanoparticles on An Au Electrode. Energy Environ. Sci. 2013, 6, 625-633.

17. Voiry, D.; Yamaguchi, H.; Li, J.; Silva, R.; Alves, D. C.; Fujita, T.; Chen, M.; Asefa, T.; Shenoy, V. B.; Eda, G. Enhanced Catalytic Activity in Strained Chemically Exfoliated $\mathrm{WS}_{2}$ Nanosheets for Hydrogen Evolution. Nat. Mater. 2013, 12, 850-855. 
18. Xiao, P.; Sk, M. A.; Thia, L.; Ge, X.; Lim, R. J.; Wang, J.-Y.; Lim, K. H.; Wang, X. Molybdenum Phosphide as An Efficient Electrocatalyst for the Hydrogen Evolution Reaction. Energy Environ. Sci. 2014, 7, 2624-2629.

19. Kong, D.; Cha, J. J.; Wang, H.; Lee, H. R.; Cui, Y. First-Row Transition Metal Dichalcogenide Catalysts for Hydrogen Evolution Reaction. Energy Environ. Sci. 2013, 6, 3553-3558.

20. McKone, J. R.; Sadtler, B. F.; Werlang, C. A.; Lewis, N. S.; Gray, H. B. Ni-Mo Nanopowders for Efficient Electrochemical Hydrogen Evolution. ACS Catal. 2013, 3, 166-169.

21. Vrubel, H.; Hu, X. Molybdenum Boride and Carbide Catalyze Hydrogen Evolution in Both Acidic and Basic Solutions. Angew. Chem. 2012, 124, 12875-12878.

22. Tran, P. D.; Nguyen, M.; Pramana, S. S.; Bhattacharjee, A.; Chiam, S. Y.; Fize, J.; Field, M. J.; Artero, V.; Wong, L. H.; Loo, J. Copper Molybdenum Sulfide: A New Efficient Electrocatalyst for Hydrogen Production from Water. Energy Environ. Sci. 2012, 5, 8912-8916.

23. Wang, D.-Y.; Gong, M.; Chou, H.-L.; Pan, C.-J.; Chen, H.-A.; Wu, Y.; Lin, M.-C.; Guan, M.; Yang, J.; Chen, C.-W. Highly Active and Stable Hybrid Catalyst of Cobalt-Doped $\mathrm{FeS}_{2}$ Nanosheets-Carbon Nanotubes for Hydrogen Evolution Reaction. Am. Chem. Soc. 2015, 137, 1587-1592.

24. $\mathrm{Xu}, \mathrm{Y}$; Wu, R.; Zhang, J.; Shi, Y.; Zhang, B. Anion-Exchange Synthesis of Nanoporous FeP Nanosheets as Electrocatalysts for Hydrogen Evolution Reaction. Chem. Commun. 2013, 49, 6656-6658.

25. Cao, B.; Veith, G. M.; Neuefeind, J. C.; Adzic, R. R.; Khalifah, P. G. Mixed Close-Packed Cobalt Molybdenum Nitrides as Non-Noble Metal Electrocatalysts for the Hydrogen Evolution Reaction. J. Am. Chem. Soc. 2013, 135, 19186-19192.

26. Zou, X.; Huang, X.; Goswami, A.; Silva, R.; Sathe, B. R.; Mikmeková, E.; Asefa, T. Cobalt-Embedded Nitrogen-Rich Carbon Nanotubes Efficiently Catalyze Hydrogen Evolution Reaction at All pH Values. Angew. Chem. 2014, 126, 4461-4465.

27. Peng, Z.; Jia, D.; Al-Enizi, A. M.; Elzatahry, A. A.; Zheng, G. Electrocatalysts: from Water Oxidation to Reduction: Homologous Ni-Co Based Nanowires as Complementary Water Splitting Electrocatalysts. Adv. Energy Mater. 2015, 5, 1402031 .

28. Liu, D.; Lu, Q.; Luo, Y.; Sun, X.; Asiri, A. M. $\mathrm{NiCo}_{2} \mathrm{~S}_{4}$ Nanowires Array as An Efficient Bifunctional Electrocatalyst for Full Water Splitting with Superior Activity. Nanoscale 2015, 7, 15122-15126.

29. Jin, H.; Wang, J.; Su, D.; Wei, Z.; Pang, Z.; Wang, Y. In Situ Cobalt-Cobalt Oxide/N-Doped Carbon Hybrids as Superior Bifunctional Electrocatalysts for Hydrogen and Oxygen Evolution. J. Am. Chem. Soc. 2015, 137, 2688-2694.

30. Stern, L.-A.; Feng, L.; Song, F.; Hu, X. $\mathrm{Ni}_{2} \mathrm{P}$ as A Janus Catalyst for Water Splitting: the Oxygen Evolution Activity of $\mathrm{Ni}_{2} \mathrm{P}$ Nanoparticles. Energy Environ. Sci. 2015, 8, 2347-2351.

31. Luo, J.; Im, J.-H.; Mayer, M. T.; Schreier, M.; Nazeeruddin, M. K.; Park, N.-G.; Tilley, S. D.; Fan, H. J.; Grätzel, M. Water Photolysis at $12.3 \%$ Efficiency via Perovskite Photovoltaics and Earth-Abundant Catalysts. Science 2014, 345, 1593-1596.

32. Wu, H. B.; Xia, B. Y.; Yu, L.; Yu, X.-Y.; Lou, X. W. D. Porous Molybdenum Carbide Nano-Octahedrons Synthesized via Confined Carburization in Metal-Organic Frameworks for Efficient Hydrogen Production. Nat. Commun. 2015, 6, 6512.

33. Yang, Y.; Fei, H.; Ruan, G.; Xiang, C.; Tour, J. M. Efficient Electrocatalytic Oxygen Evolution on Amorphous Nickel-Cobalt Binary Oxide Nanoporous Layers. ACS Nano 2014, 8, 9518-9523.

34. Shen, M.; Ruan, C.; Chen, Y.; Jiang, C.; Ai, K.; Lu, L. Covalent Entrapment of Cobalt-Iron Sulfides in N-Doped Mesoporous Carbon: Extraordinary Bifunctional Electrocatalysts for Oxygen Reduction and Evolution Reactions. ACS Appl. Mater. Interfaces 2015, 7, 1207-1218.

35. Ni, B.; Wang, X. Edge Overgrowth of Spiral Bimetallic Hydroxides Ultrathin-Nanosheets for Water Oxidation. Chem. Sci. 2015, 6, 3572-3576.

36. Liang, H.; Meng, F.; Cabán-Acevedo, M.; Li, L.; Forticaux, A.; Xiu, L.; Wang, Z.; Jin, S. Hydrothermal Continuous Flow Synthesis and Exfoliation of NiCo Layered Double Hydroxide Nanosheets for Enhanced 
Oxygen Evolution Catalysis. Nano Lett. 2015, 15, 1421-1427.

37. Li, Y.; Hasin, P.; Wu, Y. $\mathrm{Ni}_{\mathrm{x}} \mathrm{Co}_{3-\mathrm{x}} \mathrm{O}_{4}$ Nanowire Arrays for Electrocatalytic Oxygen Evolution. Adv. Mater. 2010, 22 , 1926-1929.

38. Hu, H.; Guan, B.; Xia, B.; Lou, X. W. Designed Formation of $\mathrm{Co}_{3} \mathrm{O}_{4} / \mathrm{NiCo}_{2} \mathrm{O}_{4}$ Double-Shelled Nanocages with Enhanced Pseudocapacitive and Electrocatalytic Properties. J. Am. Chem. Soc. 2015, 137, 5590-5595.

39. Landon, J.; Demeter, E.; Inoğlu, N.; Keturakis, C.; Wachs, I. E.; Vasić, R.; Frenkel, A. I.; Kitchin, J. R. Spectroscopic Characterization of Mixed Fe-Ni Oxide Electrocatalysts for the Oxygen Evolution Reaction in Alkaline Electrolytes. ACS Catal. 2012, 2, 1793-1801.

40. Liu, Y.; Cheng, H.; Lyu, M.; Fan, S.; Liu, Q.; Zhang, W.; Zhi, Y.; Wang, C.; Xiao, C.; Wei, S. Low Overpotential in Vacancy-Rich Ultrathin $\mathrm{CoSe}_{2}$ Nanosheets for Water Oxidation. J. Am. Chem. Soc. 2014, 136, 15670-15675.

41. Song, F.; Hu, X. Exfoliation of Layered Double Hydroxides for Enhanced Oxygen Evolution Catalysis. Nat. Commun. 2014, 5, 4477.

42. Jiang, N.; You, B.; Sheng, M.; Sun, Y. Electrodeposited Cobalt-Phosphorous-Derived Films as Competent Bifunctional Catalysts for Overall Water Splitting. Angew. Chem. Int. Ed. 2015, 54, 6251-6254.

43. Song, F.; Hu, X. Ultrathin Cobalt-Manganese Layered Double Hydroxide is An Efficient Oxygen Evolution Catalyst. J. Am. Chem. Soc. 2014, 136, 16481-16484. 